\title{
Linx
}

Revue des linguistes de l'université Paris X Nanterre

$46 \mid 2002$

Les connecteurs

\section{Les connecteurs hatta / même : emplois et valeurs. Perspective contrastive arabe-français}

\section{Mongi Kahloul}

\section{(2) OpenEdition}

\section{Journals}

Édition électronique

URL : http://journals.openedition.org/linx/92

DOI : $10.4000 /$ linx.92

ISSN : 2118-9692

\section{Éditeur}

Presses universitaires de Paris Nanterre

\section{Édition imprimée}

Date de publication : 1 juin 2002

Pagination : 51-67

ISSN : 0246-8743

\section{Référence électronique}

Mongi Kahloul, «Les connecteurs hatta / même : emplois et valeurs. Perspective contrastive arabe-

français », Linx [En ligne], 46 | 2002, mis en ligne le 27 janvier 2011, consulté le 19 avril 2019. URL

http://journals.openedition.org/linx/92 ; DOI : 10.4000/linx.92 


\title{
Les connecteurs hatta / même: emplois et valeurs. Perspective contrastive arabe-français.
}

\author{
Mongi Kahloul, Faculté des Lettres et des Sciences \\ humaines de Sousse
}

"مات وفي نفسه شيء من حثّى"

\section{Introduction générale}

Les recherches en pragmatique en général, et plus particulièrement celles qui sont consacrées aux connecteurs, ne cessent d'évoluer, mais également de se diversifier selon les approches prises en considération par les linguistes.

Ces deux dernières décennies ont ainsi vu un avancement considérable dans la description de ces morphèmes grammaticaux qui nous fournissent les instructions nécessaires pour construire le sens. Depuis Anscombre (1973) et son article sur même sont parues entre autres les études de C. Rossari (2000) et C. Rossari-J. Jayez (1996 à 1999). S'y ajoutent tous les travaux anglo-saxons tels Oversteegen (1997), M. B. Hansen-Mosegaard (1998), etc.

En revanche, les analyses de pragmatique comparée sont plutôt peu développées dans cette perspective de recherche et plus particulièrement sur les connecteurs. On dispose simplement pour le couple français-italien des travaux de A. Ferrari et C. Rossari (1994), C. Rossari (1994) sur la reformulation, J. Visconti (1994) sur les connecteurs conditionnels complexes, la thèse de L. Sini (1997) sur les connecteurs argumentatifs et contre-argumentatifs, et dans une perspective contrastive français-chinois de M. Hoa (1986) sur la description de Mais et son correspondant chinois Keshi et plus récemment A. Auchlin (1993) qui prend en considération quelques connecteurs comme Mais, Puisque, De toute façon et leurs correspondants chinois.

\section{L'objet de la présente étude}

Cet article propose une description du fonctionnement sémantico-pragmatique du connecteur arabe Hatta (Même) dans une perspective contrastive arabe-français. Comme nous allons le découvrir tout au long de cette étude, ce marqueur se révèle polysémique et il n'a pas toujours comme équivalent son correspondant même en français. Le corpus pris en considération repose, d'une 


\section{Mongi Kabloul}

part, sur la langue arabe classique dans la mesure où nous essayerons de passer en revue les descriptions relatives à ce connecteur fournies par les grammairiens arabes, et d'autre part, sur le dialecte arabe tunisien. C'est précisément dans cette partie que l'on parlera de batta en tant que connecteur pragmatique. Ce travail a comme ultime objectif de saisir, autant que faire se pourrait, ses propriétés sémantiques mais également pragmatiques. Il s'agira donc pour nous de préciser d'une part, le rôle et les valeurs de ce connecteur, chose qui, à notre connaissance, n'a pas été faite jusqu'ici ; et de le confronter, d'autre part, avec même quand il nous est possible de le faire vu l'éloignement génétique des deux langues prises en considération.

\section{Observations générales sur Hatta (حتى )}

Les grammairiens arabes ${ }^{1}$ présentent le marqueur comme dépendant essentiellement de son usage morphosyntaxique: dans l'ensemble des grammaires consultées, nous n'avons pas rencontré d'analyse sémantique, ni pragmatique. Toutes les théories s'accordent pour dire que le sens de Hatta est tributaire de la vocalisation ${ }^{2}$ du nom ou du verbe qui lui est postposé. Autrement dit, le nom ou le verbe postposé à Hatta conditionne le sens. En guise d'illustration, nous empruntons les énoncés suivants ${ }^{3}$.

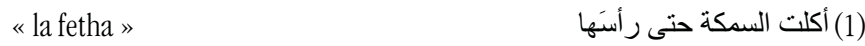

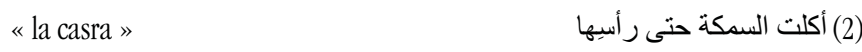

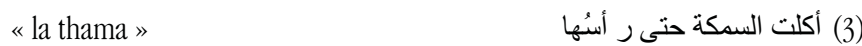

que nous traduisons par "J'ai mangé le poisson, même la tête », ou dans un français peut-être plus soutenu : «J'ai mangé le poisson, y compris la tête ». Ces énoncés n'auront pas le même sens selon que l'on vocalise le nom «tête» en [a], [i] ou [u]. Ainsi pour (1), les grammairiens arabes sont presque tous d'accord pour dire que le connecteur a la valeur de la coordination; ce qu'on pourrait gloser par : « J'ai mangé le poisson et j'ai mangé la tête aussi. »

Alors que (2), muni d'une vocalisation [i], et toutes choses égales par ailleurs, va avoir un autre sens «délimitatif » : dans cette version nous allons épargner la tête du poisson, jusqu'à se comprenant comme sauf, non compris.

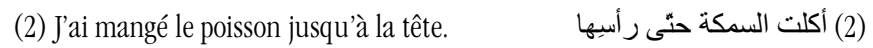

Du coup, hatta ne correspond plus à son correspondant français même. Toutes les théories confirment explicitement cet emploi précisant qu'on pourrait substituer ila (jusqu'à) à hatta:

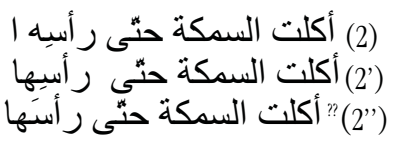

Alors que la substitution de hatta par ila ne pose aucun problème de sens à partir du moment où nous avons toujours la vocalisation en "casra », (2') et (2") ne sont pas acceptables et

\footnotetext{
${ }^{1}$ Al-kitab, kitab Sibaweih pp17.

2 La vocalisation en arabe se manifeste sous forme de petits signes qu'on met sur les lettres pour donner les timbres des voyelles. Elle se compose de trois voyelles [a], [i] et [u] qu'on appelle respectivement la fetha, la casra et la thama.

3 Ces énoncés sont prélevés de Al-moghni-1-labib pp175.
} 
même contradictoires car ila n'est pas compatible avec la « fetha ». En effet, le contenu propositionnel en (2") devient l'équivalent de deux contenus propositionnels contradictoires formulés en (a) et (a') :

$\mathrm{A}^{\circ} \quad$ J'ai mangé le poisson même la tête : $\mathrm{r}$ : (la totalité du poisson, formulé sur le mode explicite)

(a') J'ai mangé le poisson jusqu'à la tête : r : (sauf la tête, formulé sur le mode implicite)

Si, en français, il n'y a pas d'ambiguité possible entre même et jusqu'à dans l'usage et dans l'emploi, en arabe ces deux marqueurs ne font qu'un hatta. Nous en concluons, comme les grammairiens arabes, que la vocalisation est bel et bien responsable du sens de l'énoncé sans pour autant disposer d'hypothèse sur les moyens par lesquels s'effectue l'interprétation.

Nous en venons à présent à la troisième version. Le même énoncé muni d'une vocalisation en [u] (thama) nous renseigne par implicitation sur la tête qui est mangée.

A l'instar de (2") où le ila (qu'on traduit en français par jusqu'à) semble contradictoire, la même substitution dans (3) ne fait que confirmer cette contradiction.

(3) J'ai mangé le poisson, y compris la tête.

$$
\text { (3) (3) أكلت السمكة حنّى رأسُها }
$$

(3') J'ai mangé le poisson, jusqu'à la tête.

Dans la mesure où le locuteur ne peut affirmer une chose et son contraire, en l'occurrence d'avoir mangé la totalité du poisson y compris la tête et le même poisson sans la tête, (3') se révèle inacceptable.

\section{Hatta et le verbe}

Par ailleurs, les grammairiens arabes décrivent le fonctionnement de batta suivi d'un verbe. Quoique cette partie se trouve controversée, on se bornera ici à reprendre les constantes confirmées et reprises par la majorité des auteurs. En gros, il est recensé deux sens de batta suivi d'un verbe que nous traduisons respectivement par :
1- Justification

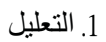
2- Restriction

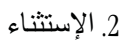

\section{Hatta à valeur de justification :}

Du point de vue formel, le hatta à valeur pragmatique de justification est toujours en position intra/interphrastique. Son rôle est de justifier et de légitimer l'action prédiquée dans le verbe principal qui précède le connecteur. Dans ce cas précis, l'appellation de « justification » est rattachée au rôle qu'assume le connecteur en fournissant la réponse à l'action contenue dans le verbe qui lui est antéposé.

Considérons l'énoncé suivant :

(4) Sois musulman si tu veux aller au paradis
(4')Sois musulman pour que tu ailles au paradis.

${ }^{4}$ Si nous mettons le présent au lieu du subjonctif c'est pour rester fidèle à la traduction littérale de la phrase arabe 


\section{Mongi Kabloul}

L'argument proposé par ces linguistes est que batta et kei sont doués illocutoirement d'une intentionnalité que ila n'a pas. A vrai dire, le mot exact utilisé pour exprimer l'idée de l'intentionnalité en arabe est le mot préméditation, que nous trouvons un peu trop fort en français et auquel nous avons préféré intentionnalité.

Force est de constater que hatta, dans cet emploi, s'éloigne assez du connecteur même en français. En effet, nous ne pouvons pas avoir :

"Sois musulman même tu iras au paradis.

Nous dirions plus spontanément : "Sois musulman pour aller au paradis » ou plutôt «Sois musulman si tu veux aller au paradis », mais on ne peut formuler l'idée à l'aide du connecteur même. L'énoncé (5) selon la substitution opérée ajoute quelques nuances de sens à cette valeur de justification :

(5) J'ai combattu afin de la libérer.

$$
\text { (5) (5اتلت حيّى أحرّز ها؟ قاتلت إلى (أن) أحرّر ها }
$$

(5') "'J'ai combattu jusqu'à/pour la libérer

L'emploi de ila, dans (5') est non attesté au sens « justification » car il véhicule non plus la force illocutoire exprimant la motivation à combattre mais plutôt l'acharnement et la persévérance intrinsèques à celle-ci, ce qu'on pourrait gloser par: "Je combattrai jusqu'à sa libération " à condition bien sûr de mettre le verbe antéposé à ila au présent de l'indicatif au lieu du passé :

(5") Je combattrai jusqu'à ce que je la libère.

$$
\text { (5) أقاتلُ إلى أن أحرّرها }
$$

Avec le verbe au passé, antéposé à ila en arabe nous aurons une contradiction dans la mesure où le procès de combattre est envisagé comme un processus duratif et non comme un procès révolu. De ce fait, nous pouvons remarquer l'inappropriété aspectuelle de ila dans cet emploi.

Avec kei et hatta nous continuons à avoir la valeur de justification en ce sens que l'on justifie le combat par la libération. En français nous sommes plutôt enclins à dire qu'il s'agit là d'une valeur de but car la traduction la plus spontanée serait : « J'ai combattu pour la libérer » ou « ... afin de la libérer ». Hatta se distingue peut-être par une nuance de sens dans la mesure où sa valeur illocutoire s'imprègne de plus de fermeté et d'obstination que celle de kei. restriction.

Accompagné du morphème discontinu de la négation ne, hatta peut avoir la valeur de

\subsection{Hatta à valeur de restriction}

Le ne...que arabe se traduit par la ou lan $+\mathrm{V}_{\text {présent }}+$ batta. Nous disions au début de cette recherche que le connecteur était polysémique et nous avons là un nouvel exemple qui s'éloigne des emplois connus et attestés pour le connecteur correspondant même car si nous devons exprimer la restriction en français, nous n'utilisons pas même mais ne que. L'énoncé: «Par Dieu je ne combattrai "même tu combattras » est agrammatical par opposition à : « Par Dieu je ne combattrai que lorsque tu combattras ». Afin de mieux visualiser cette valeur de restriction, nous procédons à la confrontation de batta dans l'énoncé (6) avec deux autres connecteurs susceptibles de suggérer les mêmes valeurs pragmatiques, dans (6') et (6") :

(6) Par Dieu, je ne combattrai que lorsque tu combattras.

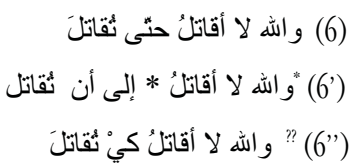

(6") Par Dieu, je ne combats pas pour que tu combattes

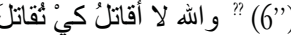


Si dans (6) l'énoncé est tout à fait acceptable en arabe et ne présente aucune ambiguïté quant au sens de la restriction, les énoncés (6') et (6") se révèlent, toutes choses étant égales par ailleurs, non naturels et en tout cas éloignés de la valeur de restriction. En effet, si dans (6) le sens est que «mon combat est, en quelque sorte, tributaire de l'action de combattre de l'interlocuteur », (6') même si elle est grammaticale est tout à fait impertinente. Quant à (6"), elle est grammaticale et tout à fait cohérente mais s'éloigne de la restriction au profit d'une autre valeur, celle du but. Autrement dit, si le remplacement de batta par ila rend l'énoncé incohérent et peu naturel dans (6'), le remplacement de batta par kei dans (6") nous donne une tout autre valeur mettant l'accent non sur « la condition de combattre » comme (6), mais sur « la raison de combattre ».

\section{Hatta avec les noms et les adjectifs}

Après les verbes, nous allons prendre en considération le comportement de ce connecteur avec les noms et les adjectifs. Les grammairiens arabes recensent deux valeurs traduites littéralement «gradabilité en plus» et «gradabilité en moins». Dans des termes plus actuels on parlera de renchérissement.

\section{1. Hatta à valeur de renchérissement}

Pour mieux comprendre ces deux valeurs, considérons les deux énoncés suivants :

(7) Les maîtres m'ont frappé, même leurs esclaves.

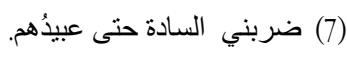

(8) Tous les gens sont mortels, même le prophète Mahomet. (8) (8) مات الناس حتى محمد ماني

Selon eux (7) serait « gradable en moins », et, (8) « gradable en plus » sans qu'ils expliquent au préalable la manière dont s'élabore cette notion de gradabilité. C'est ce que nous allons essayer de faire afin de cerner quelque peu la démarche cognitive qui sous-tend cette notion. Pour ce faire, nous serons amené à multiplier les énoncés de l'arabe, mais également du français, car, dans cette perspective les deux connecteurs hatta/même véhiculent la valeur de renchérissement.

En effet, nous pensons que l'argument présenté pour justifier la «gradabilité en moins » dans (7) est essentiellement fondé sur le lexique, dans la mesure où l'on oppose mâtres à son antonyme esclaves. D'un point de vue pragmatique, la connexion à l'aide de hatta fait ressortir précisément cette notion de «gradabilité en moins », en l'accentuant négativement. En somme, la face positive du locuteur se trouve doublement menacée : d'une part, par les mâtres et d'autre part, par les esclaves. L'interprétation véhiculée grâce au connecteur est glosable par : «non seulement je suis frappé par les maîtres mais aussi par leurs esclaves (ce qui est plus humiliant encore selon le contexte et surtout aux yeux du locuteur). En fait, la surenchère s'opère selon les échelles argumentatives de 0 . Ducrot. Si l'on compare batta avec son correspondant français même :

Les maîtres m'ont frappé, " même / et même leurs esclaves.

on observe une convergence d'usage dans la mesure où tous deux véhiculent la valeur pragmatique de «gradabilité », connue plus communément sous le nom de renchérissement. La "gradabilité en moins » s'inscrit en définitive dans une logique de degrés de menace infligés à la face positive du locuteur. Autrement dit, plus celle-ci est menacée, plus le sentiment d'humiliation est grand ; et par conséquent le renchérissement s'opère dans le sens de la flèche discontinue de notre schéma. 
Mongi Kabloul

$\left\{\begin{array}{r}\text { - maîtres } \\ \text { - esclaves }\end{array}\right.$

Quant à (8), considéré par les grammairiens arabes comme « gradable en plus », ce n'est plus le lexique qui est responsable de ce renchérissement mais plutôt l'intérêt que présente la personne même du Prophète Mahomet. En effet, la restitution s'opère par le moyen du connecteur hatta qui va embrayer sur l'énoncé pour généraliser une réalité inéluctable à tous les êtres humains, en l'occurrence «la mort». Le prophète, étant respecté par les musulmans en tant que messager de Dieu, ne devrait pas, à la limite, mourir, et c'est précisément là le foyer du renchérissement, comme pour dire: «La mort n'épargne personne, même pas Mahomet ». L'interprétation à caractère de « renchérissement en plus » sera puisée dans la hiérarchie qui va du simple musulman au messager de Dieu (le prophète). La surenchère s'opère de bas en haut selon la flèche de l'échelle de gradabilité :

$$
\left\{\begin{array}{l}
\text { - Le Prophète Mahomet est mortel. } \\
\text { - Même } \\
\text { - Tous les gens sont mortels. }
\end{array}\right.
$$

La traduction française renferme également cette valeur de renchérissement : « Tous les gens sont mortels même le prophète Mahomet ». Dans la même perspective, considérons les énoncés suivants pris de l'arabe littéral également :

(9) Ô surprise ! même Kouleib m'insulte !

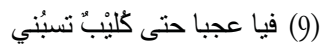

(10) Zeid est tellement malade que son état devient désespéré مرض زيد حتى إنهم لا يرجوُنَّ

En comparant (7) à (9), nous pouvons d'ores et déjà avancer l'hypothèse qu'avec le hatta «gradable en moins » nous avons la restitution d'une ellipse implicitée glosable par: «Les maitres m’ont frappé, et même leurs esclaves (m’ont frappé). La (9) serait glosée par : « Ô surprise! (les gens m'insultent) même Kouleib m'insulte ». En effet, l'ellipse dans (7) est postposée au connecteur, alors que dans la (9) elle lui est antéposée. En utilisant les variables $P$ et $Q$ nous aurons les schémas suivants :

(7) P hatta Q (ellipse)

(9) P (ellipse) hatta Q

Toutefois, dans l'énoncé (10), la « gradabilité en moins » n'est pas véhiculée par l'énonciation mais plutôt par le connecteur intraphrastique. En effet, ce dernier, ici, renchérit sur le degré de la maladie de Zeid au point de rendre impossible l'espoir de guérison. Le schéma que nous proposons montre bien l'état dégénératif de la maladie et le renchérissement est traduit par la flèche :

$$
\sqrt{ }\left[\begin{array}{l}
\text { - malade } \\
\text { - très malade } \\
\text { - état désespéré } \\
\text { - (la mort) }
\end{array}\right.
$$


Si nous inversons le sens de l'échelle c'est pour mieux suggérer cet état de dégénérescence qui, comme la flèche l'indique, va vers le pire. En fait, le sens réel de «la gradabilité en moins » tel que stipulé par les grammairiens arabes est fondé sur la gradation lexicale allant du «mal »au « pire » : tous les lexèmes de (10) s'opposent en bloc à l'état de guérison.

Pour comparer ce connecteur avec son équivalent de la langue française même, nous pouvons très bien dire dans le même contexte " Zeid est très malade, et même dans un état désespéré ». A l'instar de batta, l'opérateur même véhicule l'interprétation du renchérissement, ou de la «gradation» pour reprendre les termes de ces grammairiens. En revanche, à notre connaissance, ces derniers n'ont pas évoqué l'existence d'un autre marqueur arabe bal (بل ) jouant le rôle de renchérissement. Force est de constater que celui-ci est polysémique et qu'à la base il exprime la rectification, l'équivalent de plutôt en français. Nous allons procéder au test de la substitution dans les énoncés qui suivront afin de vérifier le rôle sémantico-pragmatique des deux connecteurs dans cette entreprise illocutionnaire de renchérissement. Pour ce faire, nous avons choisi ces énoncés où hatta opère sur des adjectifs qui lui sont postposés.

Considérons le corpus suivant :

(11) Il est intelligent, même génial.

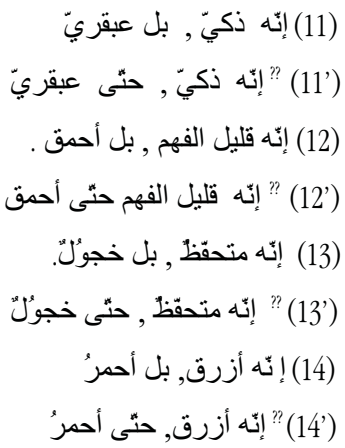

Dans les énoncés (11), (12) et (13), la valeur pragmatique est exclusivement de renchérissement, véhiculée par le connecteur bal qui augmente l'intelligence en génie dans (11), l'inintelligence en idiotie dans (12) et l'état de réservé en timidité dans (13). Dans tous les énoncés dans lesquels nous avons substitué hatta à bal, c'est-à-dire (11'), (12'), (13') et (14'), le connecteur batta sera traduit par aussi. Quant à l'énoncé (14), le connecteur reprend sa valeur primaire et se réduit en un adverbe plutôt à caractère de rectification, dans la mesure où il s'agit là de couleurs bleue et rouge et que le rouge ne peut pas être le renchérissement du bleu au même titre que « génial » qui est, en effet, le renchérissement d'« intelligent», ou de « réservé » par rapport à «timide» dans (13). Dans (14) le connecteur n'a de valeur que celle de revenir sur l'avant-plan discursif en vue de rectifier et mettre la couleur rouge à la place de la couleur bleue. Dans (14') et avec le connecteur batta, nous n'aurons plus à rectifier un énoncé comme dans (14) mais nous ajoutons tout simplement une autre couleur à celle existant déjà. Du coup, nous changeons complètement d'énoncé et hatta se transforme dans ce cas précis en une conjonction de coordination et. Pour pousser un peu plus l'analyse, et afin de confirmer la polysémie du connecteur bal, nous avons choisi comme énoncés (15) et (16). Dans la structure monologale, (15) perd cette valeur de renchérissement et devient un énoncé contradictoire. Il reprend son statut d'opérateur rectificatif dans (16). 
(15) *Il est intelligent au contraire bête.

(16) A- Il est intelligent

B- Au contraire il est bête

$$
\begin{aligned}
& \text { (15) (15) إنّه ذكيٌّ بل أحمَقُ }
\end{aligned}
$$

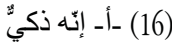

$$
\begin{aligned}
& \text { ــــ بل أحمقُ. }
\end{aligned}
$$

Selon le cadre polyphonique fixé par 0. Ducrot (1984), le connecteur bal dans (16 B) marque une opposition vis-à vis de la réplique de $\mathrm{A}$ dans la structure dialogique :

$$
\begin{aligned}
& \mathrm{Si} A \text { dit que } \mathrm{P} \\
& \mathrm{B} \text { soutient que non } \mathrm{P} \Rightarrow \text { mais } \mathrm{q}
\end{aligned}
$$

Si nous refusons en (15) la valeur pragmatique de renchérissement véhiculé par bal, c'est que dans la structure monologale, le même locuteur ne peut soutenir à la fois la chose et son contraire, ce qui serait glosable en français par « Il est intelligent au contraire il est bête » pas même si l'on accorde à bal le sens de plutôt : «Il est intelligent plutôt bête » à moins de considérer une catégorie d'intelligents qui serait appelée « les intelligents plutôt bêtes » ; pourquoi alors avons-nous accepté dans (14) le même connecteur quand il s'agissait des couleurs bleue et rouge? On peut avancer l'hypothèse que c'est peutêtre parce qu'il n'existe pas de relation antonymique entre ces adjectifs de couleur alors qu'elle existe bien entre «intelligent» et «bête» dans (15) : le connecteur bal adversatif ne serait opérationnel qu'avec les lexèmes entretenant un rapport antonymique et il n'aurait de valeur de renchérissement que lorsqu'il existe une gradation dans le sémantisme des items en question.

Par conséquent, le bal adversatif aurait comme correspondant en français le connecteur au contraire dans la structure dialogique, et le connecteur même quand il s'agit de renchérissement dans la structure monologale. En effet, un énoncé du type « Jean est réservé, (et) même timide » est tout à fait acceptable en français. En revanche, la substitution de bal à hatta dans (17) ne présente aucun intérêt dans la mesure où elle aboutit à un énoncé étrange et peu naturel en arabe. Il est intéressant de noter que le marqueur même en français se révèle aussi étrange et forme un énoncé complètement saugrenu voire contradictoire et ce, dans les deux structures monologale et dialogique, notées respectivement (17) et (17').

(17) *Il est intelligent, même bête

(17') A : Il est intelligent. B : * (et) même bête.

$$
\begin{aligned}
& \text { (17) إنّه ذكيّ , بل أحمق }
\end{aligned}
$$

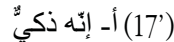

$$
\begin{aligned}
& \text { ب- * حنّى أحمق }
\end{aligned}
$$

Le connecteur même dans ces occurrences n'est pas opérationnel car, comme nous l'avons précisé plus haut, il n'existe pas de gradation dans le sémantisme des lexèmes pris en considération dans la mesure où « bête » n'est pas le renchérissement d' « intelligent ».

\subsection{Hatta à valeur méronymique 5 (partie-tout).}

Cette partie constitue la description d'une autre valeur assignée au connecteur hatta. A l'aide d'énoncés empruntés à Ibnu Hicham, nous allons analyser mais également expliciter la valeur méronymique contenue dans ce corpus.

Les énoncés (18), (19) et (20) illustrent donc la valeur dite «méronymique », connue également sous la terminologie de « relation partie-tout».

(18)Les pèlerins sont revenus, même les piétons d'entre eux. قدمَ الحجيج حنى المشاةُ (19)

(19) La servante m'a plu, (et) même son discours. اعجبتني الجارية حتى حديثهائ (19)

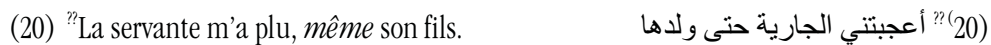

\footnotetext{
5 Terme proposé par D. A. Cruse (1986), Lexical semantics, Cambridge University Press.
} 
Ibnu Hicham stipule qu'il existe un test pour vérifier cette valeur méronymique, qui est celui de la restriction ne...que. Soit lam (ل) le correspondant syntaxique du morphème discontinu de la négation $n e$ et illa ${ }^{6}$ (إل) ce qui serait en français l'équivalent de la conjonction que:

$$
\begin{aligned}
& \text { (18')Ne sont revenus de la Mecque que les piétons. } \\
& \text { (18') لم يقدم من الحجيج إلاّ المشاةُ } \\
& \text { (19')Ne m'a plu chez la servante que son discours. } \\
& \left(20^{\prime}\right)^{*} \mathrm{Ne} \text { m'a plu en la servante que son fils. }
\end{aligned}
$$

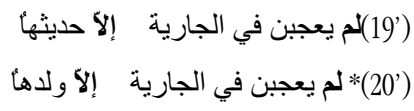

Nous comprenons aisément, par processus inférentiel, que le lexème «piétons ${ }^{7}$ » dans (18) renvoie sans ambiguité aux pèlerins qui ont voyagé à pied. Nous aurons par là même l'interprétation méronymique dans la mesure où nous comprenons que c'est une partie de la totalité des pèlerins qui est revenue. De même dans (19') il s'agit spécifiquement du discours de la servante et par conséquent « discours » est intimement lié à « servante ». En revanche, dans (20') le test de restriction n'est plus concluant, dans la mesure où l'énoncé restrictif «*Ne m'a plu en la servante que son fils » ne constitue pas un énoncé acceptable de la langue arabe, de fait, la phrase ne renferme aucune valeur méronymique (dans la mesure où le fils n'est pas une partie, physiquement en tout cas, de la mère), ce qui confirme que le connecteur hatta véhicule bel et bien la valeur méronymique dans les énoncés (18) et (19).

Voyons à présent si le marqueur français même est en mesure de suggérer à son tour cette valeur. Pour ce faire, considérons les énoncés :

(21) Ils ont bombardé le village, même l'église.

(22) Pierre a tout réparé sur sa voiture, même les essuie-glaces.

(23) 'Tout me plaît chez lui, même sa cravate.

(24) Tout me plaît chez lui, même son nez crochu.

Le test de ne...que s'avère pertinent :

(21') Ils n'ont bombardé du village que l'église.

(22') Pierre n'a réparé sur sa voiture que les essuie-glaces.

$\left(23^{\prime}\right)^{?}$ Ne me plait chez lui que sa cravate.

(24') Ne me plaît chez lui que son nez crochu.

En effet, nous pouvons dire que même comme la restriction sont valables excepté en (23): l'église fait bien partie du village dans (21), les essuie-glaces sont bien une partie également de la voiture, comme le nez est une partie du corps du délocuteur en question. En revanche, en (23), même si l'énoncé est acceptable en français, nous l'avons doté d'un point d'interrogation pour souligner le fait que la cravate ne fait pas partie de la personne qui la porte, du moins, au même titre que l'église dans (21) ou des essuie-glaces dans (22). Cet énoncé nous rappelle un peu (20) où le fils n'est pas non plus une partie de la mère. Nous pouvons donc affirmer qu'il s'agit là d'une valeur commune aux deux langues arabe et française.

\footnotetext{
${ }^{6}$ Nous transcrivons délibérément illa avec deux consonnes [1] d'abord pour le différencier du connecteur ila qui se traduit en français par jusqu'à et ensuite pour mettre en exergue la géminée arabe.

7 Faute de mieux, nous avons opté pour le terme «piétons » pour désigner les pèlerins marcheurs et les distinguer de ceux qui se rendent par voie aérienne ou autres.
} 


\section{Mongi Kabloul}

Après ces analyses consacrées à batta dans la langue littérale arabe, nous passons à l'examen de quelques occurrences dialectales afin de cerner davantage le rôle sémantico-pragmatique que revêt le connecteur.

\section{Hatta et l'arabe dialectal tunisien}

L'étude concernant l'emploi de hatta dans le dialecte tunisien se veut un complément de celle de la langue classique. A notre connaissance, ce marqueur n'a jamais fait l'objet de quelque analyse pragmatique auparavant. Nous précisons également que le corpus pris en considération est authentique et extrait de la vie quotidienne. Nous avons pu recenser trois valeurs à ce connecteur : la première est à caractère « délimitatif » et hatta se traduit littéralement par jusqu'à en français, les deux autres s'inscrivent totalement dans la pragmatique et ont trait à l'« ironie » d'une part, et à la «menace » d'autre part.

\subsection{Hatta le délimitatif ${ }^{8}$ :}

Dans un premier emploi, ce connecteur est le plus souvent traduit, comme nous l'avons mentionné plus haut, par jusqu'à en français et ne peut, toutes choses étant égales par ailleurs, être traduit par même. L'énoncé (25) dans la structure de l'échange illustre cette valeur :

(25) A-T'en es où dans le roman?

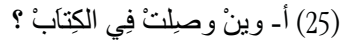

$$
\begin{aligned}
& \text { بـ قريتُ حتى صفحة } 111 .
\end{aligned}
$$$$
\text { B-j'ai lu jusqu'à la page } 111 .
$$

L'opérateur hatta délimite, dans la réplique de B, les pages lues et jalonne pour le lecteur l'arrêt de la lecture. Nous ne voyons pas de valeur possible équivalente au correspondant même, sinon peut-être, et à condition de changer de contexte, ce que nous pouvons gloser en français par « J'ai même lu la page 111. », ou encore "J'ai lu même la page 111. ». Or, dans cette dyade, et selon la question de A, considérer hatta au sens de même nous paraît, pour le moins, inapproprié, voire injustifié dans la mesure où l'interlocuteur verra d'un œil curieux l'usage du connecteur ici, sachant que la page 111 n'a rien de particulier par rapport aux autres pages du livre en question. En revanche, et pour légitimer cet usage, il faudrait trouver un contexte dans lequel même s'érige en un « intensificateur » ou un «focalisateur » ${ }^{9}$ qui renchérit sur l'importance de la page en question. Nous pouvons, pour faciliter l'accès à cette interprétation d'intensificateur, imaginer un contexte dans lequel la page 111 serait par exemple difficile à lire, incohérente ou alors, et dans un contexte ironique, une page manquante dans ce livre. En somme, il faudrait trouver une raison qui pourrait justifier et valider l'usage de même pour qu'il soit pertinent dans ce contexte. Or, dans (25) nous avons plutôt le schéma d'un stimulus-réponse tout à fait anodin et sans importance particulière.

\subsection{Hatta à valeur illocutoire d'ironie.}

Il existe par ailleurs une valeur ironique et de moquerie très prononcée dans le dialecte tunisien, notamment quand nous voulons tourner en dérision un objet ou une personne, valeur décelable aussi bien dans des structures monologales que dialogiques. En effet, le calcul interprétatif

\footnotetext{
${ }^{8}$ Le terme est fabriqué par nos propres soins pour les besoins de l'analyse.

${ }^{9}$ Ces termes sont empruntés à L. Sini (1997) dans sa thèse sur les connecteurs argumentatifs et contreargumentatifs.
} 
véhiculant l'ironie se donne à construire par l'interlocuteur. L'autre manière pour y accéder est de faire un constat émanant non plus du co-énonciateur mais de l'énonciateur, auteur de la réplique ironisante. En d'autres termes, et pour mieux expliciter le rôle pragmatique du connecteur, l'énonciateur demande à statuer sur la valeur, au sens premier, de l'objet ou de la personne dont il est question, en vue de les disqualifier. Cette entreprise sémantico-pragmatique s'inscrit pleinement dans l'illocutoire.

Trois paramètres participent, dans une large mesure, à instituer l'interprétation ironique :

1. syntaxique : Le connecteur hatta occupe toujours la position initiale dans la phrase. Celle-ci est toujours de type exclamatif.

2. phonétique : Généralement, l'énoncé contenant hatta est prononcé avec un débit plutôt lent traînant un peu sur les syllabes. Quant à l'intonation, elle est descendante et éventuellement accompagnée d'un mouvement circulaire lent de la tête.

3. pragmatique : Le rôle de hatta est de souligner ce qu'il y a de déplacé entre l'avant-plan discursif et la réplique émise par le locuteur. En effet, par la simple énonciation de l'énoncé renfermant le connecteur, le locuteur accomplit un acte illocutoire ironique et moqueur ayant pour but d'atteindre le délocuteur, qu'il soit objet ou humain, en le disqualifiant aux yeux de l'auditoire au sens générique du terme.

Pour ce faire, considérons le corpus suivant :
(26) "? Même celle-ci est actrice !
(26) (27) حتّى هذه ممنّلة
(27) "Même celui-ci est médecin!

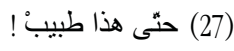
(28) "' Même lui est un homme !

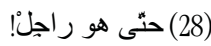
(29) "Même toi entrâneur !
(29) حنّى إنْتِ ممرَّنْ

Avant l'analyse à proprement parler de ce corpus, pour mieux situer les énoncés et enfin pour expliciter les actes illocutoires inhérents à ces occurrences, nous fournissons les contextes dans lesquels nous avons prélevé ces séquences : (26) est un commentaire à propos d'une actrice devant le petit écran, (27) en est un autre fait dans un hôpital sur un médecin, (28) est la réplique d'une femme suite à un malentendu avec son époux et (29) l'exclamation d'un joueur remettant en cause la compétence de son entraîneur.

Force est de croire que hatta dans ces énoncés est le responsable, à part entière, de cette valeur ironique-disqualifiante dans la mesure où il embraye sur la situation de l'énonciation pour assurer sa tâche de connexion et c'est la seule valeur qui se dégage de ce corpus. Afin d'y entrevoir une quelconque convergence avec le connecteur français même, nous allons traduire ces énoncés dans un français plus spontané :
(26) Tu parles d'une actrice!
(27)Tu parles d'un médecin!
(28)Tu parles d'un homme!
(29)Tu parles d'un entraîneur !

La locution tu parles apparaît être à la langue française ce que hatta est à la langue arabe. Les deux marqueurs partagent cette dimension illocutoire métacommunicative suggérant l'ironie mais aussi, dans une certaine mesure, le rejet de la vérité du contenu propositionnel inhérent à l'énoncé. L'embrayage sur la situation d'énonciation se marque formellement par le fait que dans 


\section{Mongi Kabloul}

tous les énoncés hatta est suivi soit par un démonstratif, soit par un pronom personnel. Or leur omission (premier test) est impossible, celle de batta n'étant en revanche pas totalement exclue (deuxième test). La série (26'), (27'), (28') et (29') constitue les énoncés relatifs au premier test, et la série (26"), (27"), (28") et (29") celle des énoncés relatifs au second test.

$$
\begin{aligned}
& \left(26^{\prime}\right) * \text { Même Ø actrice! } \\
& \left(27^{\prime}\right) * \text { Même Ø médecin! } \\
& \left(28^{\prime}\right) * \text { Même Ø homme! } \\
& \left(29^{\prime}\right) * \text { Même Ø entraîneur ! }
\end{aligned}
$$

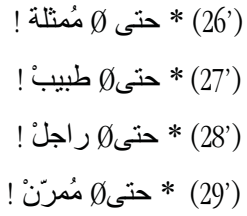

$\mathrm{Au}$ vu du premier test, force est de constater que, curieusement, nous n'avons plus de valeur suggérant l'ironie: du coup hatta se transforme en l'équivalent de l'adjectif aucun en français et le sens devient négatif. Autrement dit, l'omission du démonstratif en arabe neutralise cette dimension ironique et rend les énoncés négatifs. La traduction littérale nous donne :

\section{(26') Aucune actrice ! \\ (27’) Aucun médecin! \\ (28') Aucun homme ! \\ (29') Aucun entraîneur !}

En revanche, dans la langue française, même fournit à son tour une autre valeur de ce connecteur pour désigner «le même ». On peut d'ores et déjà avancer l'hypothèse que l'omission du démonstratif se révèle rédhibitoire quant à la valeur ironique. En effet, pour qu'il y ait ironie il faut que le connecteur arabe soit suivi par un démonstratif ou, éventuellement, par un pronom personnel.

Voyons à présent le comportement du corpus par rapport au second test :

$$
\begin{aligned}
& \text { (26") "? Ø C'est une actrice ! } \\
& \text { (27") "? Ø C'est un médecin! } \\
& \left(28^{\prime \prime}\right) \text { "? Ø C'est un homme ! } \\
& \text { (29") "' Ø Tu es entraîneur ! }
\end{aligned}
$$$$
\text { (26") }
$$

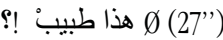$$
\text { ه (28") }
$$

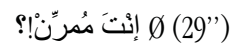

Malgré l'omission du connecteur responsable de cette valeur ironique, les énoncés produits avec une intonation descendante et un débit lent, et éventuellement accompagnés d'un mouvement circulaire de la tête, conservent quand même leur dimension sarcastique à valeur dépréciative dans le corpus arabe. Ces effets prosodiques se révèlent essentiels quant à la restitution de cette ironie. En revanche, si l'on dépouille ces énoncés de leurs caractéristiques supra-segmentales, avec une intonation plate, ils seront réduits à de simples assertions que nous traduisons respectivement par :

$$
\begin{aligned}
& \text { - C'est une actrice. } \\
& \text { - C'est un médecin. } \\
& \text { - C'est un homme. } \\
& \text { - Tu es entraîneur. }
\end{aligned}
$$

Avant de passer à l'étude finale, il nous semble que nous pouvons conclure sur les deux valeurs étudiées jusqu'à maintenant afin de les rapprocher. En effet, on a vu en 4.1. une valeur « délimitative » indiquant que nous arrivons à un terme, une limite, sans qu'ils soient inclus dans le 
procès ; ainsi en (25), la réplique B signifie « J'ai lu jusquià la page 111 non comprise », il en va de même pour (2) qui s'interprète comme «J'ai mangé le poisson jusqu'à la tête (que je n'ai pas mangée) ». De même, la valeur ironique étudiée en 4.2. vient de ce que l'énoncé met en doute la pleine réalisation de la qualité : (26) signifie que la comédienne en question n'est pas tout à fait une actrice, qu'elle n'atteint pas le stade où l'on pourrait vraiment parler d'« actrice » à son propos. Dans les deux cas (4.1 et 4.2) batta exprime donc le fait que le procès ou l'état n'inclut pas le terme exprimé, qu'il n'aboutit pas à la frontière attendue (je n'ai pas lu la page 111, du moins dans sa totalité, et cette comédienne que je vois n'est pas vraiment digne de ce nom).

Nous en venons enfin à la dernière valeur illocutionnaire de hatta dans l'arabe dialectal tunisien. Celle-ci est traduite dans des termes à caractère de menace. Elle est proactive dans la mesure où le procès est envisagé dans le futur proche ou lointain.

\subsection{Hatta à valeur illocutoire de menace.}

Avant d'entreprendre l'analyse, précisons les contextes des énoncés sur lesquels nous allons travailler : (30) constitue les propos d'une mère menaçant son fils qui s'enfuit après avoir cassé un vase, et (31) a été entendu lors d'une querelle dans une cour d'école.

(30) * Même je t'attrape entre mes mains!

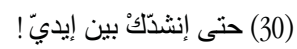

(31) * Même tu verras ce que je te ferai !

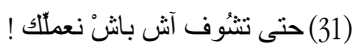

Les paramètres essentiels pour véhiculer cette valeur de menace sont les suivants :

- du point de vue syntaxique batta occupe, comme le précédent, la position initiale de la phrase. Celle-ci est de type exclamatif.

- du point de vue phonétique, étant donné l'état de colère, ou en tout cas d'énervement, le débit avec lequel est produit l'énoncé ne peut être que rapide et la courbe mélodique est plutôt ascendante. Comme gesticulation, l'acte de menace s'accompagne souvent d'un mouvement perpendiculaire au sol du bras droit, en serrant le pouce contre l'index.

- du point de vue pragmatique, hatta s'avère, en définitive, le connecteur qui suggère la force illocutoire inhérente à cet acte de menace. Nous avons là aussi une valeur métacommunicative embrayant sur la situation de l'énonciation.

La traduction littérale de ces deux énoncés, en gardant le connecteur en position initiale, nous donne des phrases agrammaticales et inacceptables en français (cf 30 et 31). En effet, nous avons l'impression que même, dans cette perspective pragmatique, ne présente aucun intérêt et qu'à la limite son omission n'affecte pas le contenu propositionnel de l'énoncé et réduit les énoncés en de simples assertions.

Dans un français courant spontané, nous dirions :

(30) Attends que je t'attrape !

(31) (Attends) Tu verras ce que je te ferai!

Force est de constater qu'en arabe, l'omission de hatta réduit aussi les énoncés en de simples assertions et neutralise totalement la force illocutoire de menace.

En puisant un peu plus dans le dialecte tunisien, nous avons pu trouver un autre morphème capable d'assurer la même tâche illocutionnaire et, de surcroît, avec la même valeur. Ce connecteur, pouvant se substituer parfaitement à hatta sans dénaturer, d'aucune manière que ce soit, cette 
teneur pragmatique, est le déictique temporel tawwa (maintenant). Cet adverbe de temps peut s'ériger, dans un contexte illocutionnaire donné, en un « vecteur » énonciatif suggérant la menace :

$$
\begin{aligned}
& \left(30^{\prime}\right)^{\text {? }} \text { Maintenant je t'attrape entre mes mains. }
\end{aligned}
$$

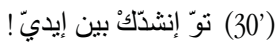

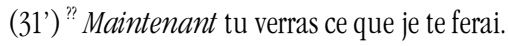

$$
\begin{aligned}
& \text { (31') }
\end{aligned}
$$

Si dans la traduction française nous respectons l'ordre des mots des énoncés arabes c'est justement pour montrer l'inadéquation du connecteur en français maintenant. En effet, ce dernier ne se révèle d'aucune utilité pragmatique pour véhiculer la menace. En revanche, cette substitution montre que les deux connecteurs arabes hatta et tawwa sont complètement interchangeables sans aucune incidence sur la valeur en question. Du coup, nous nous rendons compte qu'ils ont aussi en commun la valeur ironique (cf 4.2.) comme le montre la substitution à hatta de tawwa :

(32)" Maintenant celle-ci est actrice !?

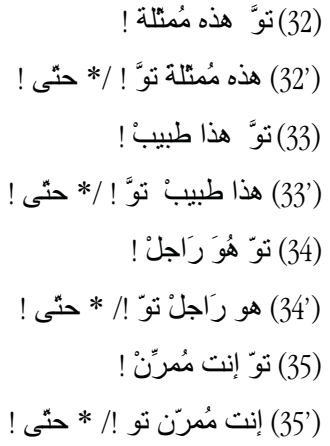

(32') Celle-ci est actrice maintenant !?

(33) "Maintenant celui-ci est médecin !?

(33’) Celui-ci est médecin maintenant !?

(34) "Maintenant il est homme!?

$\left(34{ }^{\prime}\right)^{?}$ Il est homme maintenant !?

(35) "' Maintenant toi entraîneur!?

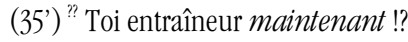

Non seulement le marqueur tawwa réussit à véhiculer la même valeur de menace que hatta, mais il le fait avec une souplesse dans l'emploi que n'a pas son concurrent comme nous le voyons dans (32'), (33'), (34') et (35'). En effet, tawwa peut occuper aussi bien la place initiale de l'énoncé que la finale. En revanche, hatta se révèle moins souple et ne peut occuper que la position frontale.

Quant à l'interprétation de l'ironie, nous estimons qu'elle est suggérée plus explicitement, et d'une manière plus franche, avec le connecteur hatta qu'avec tawwa, dans ses deux distributions initiale et finale. En guise d'explication, nous disons qu'avec le premier (batta) le contenu propositionnel véhiculant l'ironie émane du locuteur même et sur le mode explicite. Cependant, et avec le second (tawwa), le contenu propositionnel est d'une certaine manière donné à être confirmé par l'interlocuteur. Autrement dit, et à l'aide de ce dernier, l'énonciateur propose un contenu jugé moqueur et invite, dans une certaine mesure, le destinataire à statuer sur la légitimité de ce contenu, et de surcroît, le somme à partager avec lui cette attitude ironique. Ce qui nous permet d'ajouter que la différence entre ces deux marqueurs est la suivante: si, en tant que locuteur, je donne à l'interlocuteur l'occasion de statuer sur mes dires et de formuler son avis, c'est que, a priori, je ne suis pas si convaincu que cela de la véracité de mes propos ; tawwa soumet l'interprétation ironique à l'allocutaire comme pour lui dire : «Rassure-moi, tu penses aussi que c'est ironique ». Il faut ajouter que le locuteur laisse en suspens sa fausse question car, en réalité, il ne s'attend pas vraiment à une réponse ni à une quelconque confirmation du co-énonciateur. En revanche, avec hatta, on a affaire à un contenu propositionnel dans lequel le locuteur se prononce explicitement sur le délocuteur à ridiculiser sans pour autant impliquer d'aucune manière que ce soit l'interlocuteur. En somme, il s'agit d'un jugement personnel et pleinement assumé par l'énonciateur, auteur de la réplique. 


\section{Conclusion}

Après l'étude menée tout au long de cet article, nous sommes à même d'émettre quelques hypothèses concernant le rôle pragmatique de hatta avec son correspondant même.

Hatta selon la vocalisation est susceptible d'indiquer des valeurs contradictoires puisque

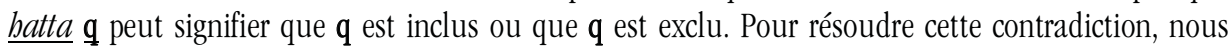
postulons que hatta introduit un terme, une limite de procès, en l'occurrence « la tête de poisson que je mange », mais sans spécifier en soi s'ils sont compris dans le procès (puisque q peut-être inclus, au sens de « y compris », ou exclu, au sens de « sauf » ou « jusqu'à » au sens de (non compris)).

Pour confirmer cette hypothèse, nous faisons le lien avec l'emploi en 2.1. où batta introduit aussi un terme à atteindre et une condition à remplir (cf (4)) dans « Sois musulman si tu veux aller au paradis ». En somme, c'est le fait de se convertir à l'Islam qui justifie le fait d'aller au paradis. L'emploi en 2.2. vient étayer cette hypothèse dans la mesure où (6) introduit également ce terme et cette condition «Je ne combattrai que si tu combattras ».

Ces deux valeurs ne sont pas partagées par le connecteur même en français. En revanche, dans 3.1. (cf (7)) nous trouvons que la valeur dite de renchérissement est tout à fait attestée dans la langue française car nous y retrouvons la même « oscillation » entre le plus et le moins. L'autre point de convergence entre les deux connecteurs se manifeste dans la valeur méronymique 3.2. (cf (18)) qui, traduit en français, ne pose aucune ambiguité quant à l'interprétation partie-tout.

En 4.2., et dans l'arabe dialectal, nous pouvons affirmer, après les substitutions syntagmatiques et paradigmatiques opérées sur batta , que, pour qu'il y ait ironie, il faut que l'énoncé comportant le connecteur ait la structure suivante :

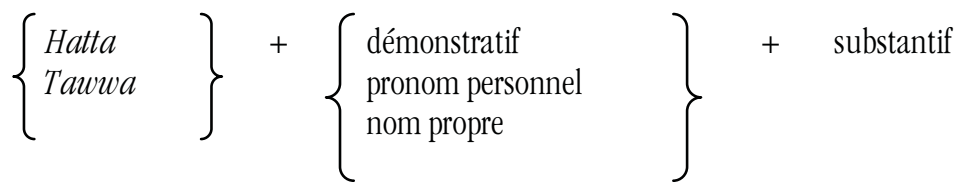

Enfin, nous pouvons dire que hatta dans 4.3. (cf (10)) exprime également que le procès n'inclut pas le terme exprimé, c'est-à-dire n'aboutit pas à la frontière attendue car dans « Attends que je t'attrape » le locuteur n'a pas encore attrapé son interlocuteur. Si même en français peut, dans des contextes bien précis, véhiculer l'ironie, il ne pourra pas pour autant véhiculer la valeur dite de menace.

(1) [akaltu samakata hatta rasaha]

(2) [akaltu samakata hatta rasiha]

(3) [akaltu samakata hatta rasuha]

(4) [aslim hatta tadkula laznatu]

(5) [qataltu hatta uharriraha]

(6) [wallahi lâ uqâtilu hatta tuqâtila]

(7) [darabani assadatu hatta abîduhum]

(8) [mâta annasu hatta muhamadun]

(9) [faya azaban hatta kuleib ta subuni]

(10)[marida zeid hatta innahum lâ jarzunahu]

(11)[innahu thakîium, bal abqarijun]

\section{CORPUS}

(12) [innahu qalîlulfahmi, bal ahmaqu]

(13) [innahu mutahaffidun, bal kazûlun]

(14) [innahu azraqu, bal ahmaru]

(15) *[innahu thakijun bal ahmaqu $]$

(16) A[innahu thakijun] $\mathrm{B}$ [bal ahmaqu ]

(17)*[innahu thakiju, bal ahmaqu ]

(18) [qadima-l-hazizu hatta-l-muchatu]

(19) [azabatni-l-zarijatu hatta hadithuha]

(20)??[azabatni-l-zarijatu hatta waladuha]

(21) à (24) sont en fançais. 
(25) A[wîn wsalt filkitab] $\mathrm{B}$ [qrît hatta safhat 111]

(26) [hatta hathi mumathla]

(27) [hatta hatha tabîb]

(28) [hatta huwa râzil]

(29) [hatta inta râzil]

(1) أكلت السمكة حتى رأسنها

(2) أكلت السمكة حتى رأسِيها

(3) أكلت السمكة حتى ر أسئها

(5) أسلم حتى تدخل الجنّة

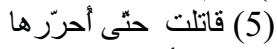

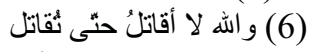

(7) ضربني السادة حتى عبيدُهم

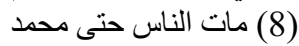

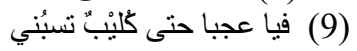

(10) مرض زيد حتى إنهم لا يرجوُنَّه

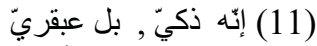

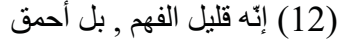

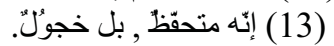

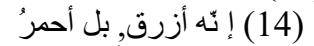

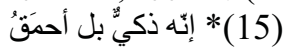

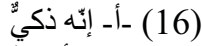

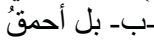

(17) * إنه ذكيّ , بل أحمق احمق
(30) [hatta inchiddik bîn îdaya]

(31) [hatta tchuf âch bâch nâmalek]

(32) [tawwa hathi mumathla]

(33) [tawwa hatha tabîb]

(34) [tawwa huwa râzil]

(35) [tawwa inta mumarrin]

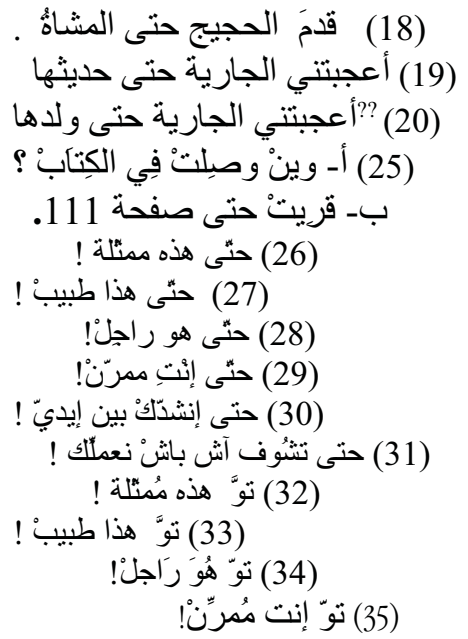

Mongi KAHLOUL

Faculté des Lettres de Sousse

(Tunisie). 
Les connecteurs hatta / même : emplois et valeurs. Perspective contrastive arabe-français

\section{BIBLIOGRAPHIE}

ANSCOMBRE J.C. (1973), « Même le roi de France est sage », Communications 20, pp 40-82.

AUCHLIN A. (1993), Faire, montrer, dire: pragmatique comparée de l'énonciation en français et en chinois, Peter Lang.

DUCROT 0. (1984), Le dire et le dit. Les éditions de Minuit.

FERRARI A. et ROSSARI C. (1994), «De donc à dunque et quindi: les connexions par raisonnement inférentiel », Cahiers de Linguistique française $n^{\circ} 15$, pp 7-49.

HANSEN-MOSEGAARD M.B. (1998), The function of discourse particles. A study with special reference to spoken standard French, Amsterdam / Philadelphia : John Benjamins.

HOA M. (1986), «Connecteurs adversatifs mais et keshi », Cahiers de Linguistique Asie Orientale n¹5, pp 65105.

OVERSTEEGEN L.E. (1997), "On the pragmatic nature of causal and contrastive connectives", Discourse Processes, 24 1, pp 51-85.

ROSSARI C. (1994), Les opérations de la reformulation: analyse du processus et des marques dans une perspective contrastive français-italien, Berne, Peter Lang.

ROSSARI C. (2000), Connecteurs et relations de discours : des liens entre cognition et signification, Presses Universitaires de Nancy.

ROSSARI C. et JAYEZ J. (1996), «Donc et les consécutifs. Des systèmes de contraintes différentiels », Linguisticae Investigationes XXI, pp 117-143.

ROSSARI C. et JAYEZ J. (1997), « Connecteurs de conséquence et portée sémantique », Cabiers de Linguistique française $\mathrm{n}^{\circ} 19$, pp 233-265.

ROSSARI C. et JAYEZ J. (1999), «Par exemple: une procédure d'exemplication par la preuve », Actes du colloque de Metz 18-20 mars 1999, Paris.

SINI L. (1997), Connecteurs argumentatifs et contre argumentatifs. Perspective contrastive français-italien, Sorbonne Nouvelle, Paris 3.

VISCONTI J. (1994), «Entre hypothèses et conditions. Sur la sémantique des connecteurs conditionnels complexes », Cahiers de Linguistique Française n ${ }^{\circ} 15$, pp 78-107.

\section{BIBLIOGRAPHIE ARABE}

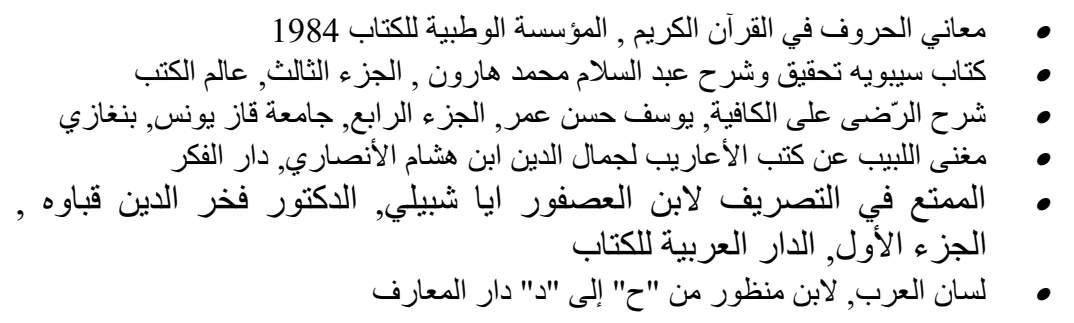


